\title{
More convenient proteasome inhibition for improved outcomes
}

The development of proteasome inhibitors for the treatment of multiple myeloma has represented a step-change for patients with this incurable plasma cell cancer, bringing increased treatment responses, and improved survival. Gradual familiarisation with toxicity profiles, and optimisation of dosing schedules, have shaped the current use of proteasome inhibitors as key components of several treatment regimens. The first-in-class proteasome inhibitor, bortezomib, was approved in 2003 for relapsed and subsequently also for newly diagnosed multiple myeloma. When used in a twice-weekly intravenous regimen, it was associated with high rates of painful and often debilitating peripheral neuropathy leading, in many patients, to treatment discontinuation. Subcutaneous administration and schedule adaptation to once-weekly use have meanwhile been shown to be both more tolerable and highly efficacious. ${ }^{1-3}$

Carfilzomib is a second-in-class epoxyketone-based, irreversibly binding, proteasome inhibitor, whereas the boronic acid-based compounds, bortezomib and ixazomib, bind reversibly, resulting in some differences in proteasomal subunit inhibition. A consecutive-day, twice weekly dosing schedule was utilised early in the development programme of carfilzomib, which was first approved by the FDA in 2012 as a single agent in relapsed disease at a dose of $27 \mathrm{mg} / \mathrm{m}^{2}$. Recently, carfilzomib was also approved in combination with dexamethasone, or lenalidomide plus dexamethasone, in the US and the European Union, based on positive results from two landmark studies, ENDEAVOR and ASPIRE. ${ }^{4-6}$ Carfilzomib dose was $27 \mathrm{mg} / \mathrm{m}^{2}$ in ASPIRE (combination with lenalidomide and dexamethasone) and $56 \mathrm{mg} / \mathrm{m}^{2}$ in ENDEAVOR (combination with dexamethasone only), using a twice-weekly consecutive-day intravenous carfilzomib infusion schedule in both studies. While twiceweekly carfilzomib at these doses is generally well tolerated, with a low rate of peripheral neuropathy, dyspnoea, hypertension and cardiac toxicities stand out as clinically relevant side effects. Moreover, in an age where the long-term prognosis continues to improve for patients with multiple myeloma, the inconvenience of a twice weekly intravenous infusion is a significant burden for both patients and healthcare providers.

In The Lancet Oncology, Philippe Moreau and colleagues ${ }^{7}$ report the results of a randomised Phase 3 study, A.R.R.O.W., in which patients who had relapsed following two or three prior treatment lines were randomised to receive carfilzomib bi-weekly at $27 \mathrm{mg} / \mathrm{m}^{2}$ or onceweekly at $70 \mathrm{mg} / \mathrm{m}^{2}$, based on a previously established maximum tolerated once-weekly dose. ${ }^{8}$ Both arms also contained weekly dexamethasone. The reported pre-planned interim analysis of the 478 patients demonstrates superior progression free survival (hazard ratio 0.69 [95\% confidence interval, 0.54-0.83]; $p=0.0029)$, as well as increased overall responses $(62.9 \%$ versus $40.8 \%$, hazard ratio 2.49 [95\% confidence interval, 1.72-3.60]; $\mathrm{p}<0.0001$ ) and deeper responses (very good partial response or better, $34.2 \%$ versus $13.4 \%$ ) with the once-weekly schedule, without any new safety signals. With the availability of different dose-scheduling regimens, considerations of safety profile are paramount. There were more $\geq$ grade 3 adverse events in the once-weekly arm (67.6\% versus $61 \cdot 7 \%)$, including sepsis and lung infections, and more frequent dose reductions, although adverse event-related treatment discontinuations of carfilzomib were similar between the arms. These differences indicate that some spectrum of toxicities may be more prominent in the once-weekly regimen, and it would be important 
to know if the adverse event profile was different in older patients; however, only a small proportion of patients in the study were older than 75 years.

The study by Moreau and colleagues will lead to the welcome availability of a more convenient once-weekly dosing schedule, and represents an important milestone for future research employing once-weekly carfilzomib in multi-agent protocols, which are becoming the standard of care for treating multiple myeloma. Some questions, however, remain to be answered, such as whether once-weekly carfilzomib is also linked to improved overall survival. Moreover, based on ENDEAVOR, the current standard dose for bi-weekly carfilzomib with dexamethasone is $56 \mathrm{mg} / \mathrm{m}^{2}$, and it remains to be established how this compares with a dose of $70 \mathrm{mg} / \mathrm{m}^{2}$ once-weekly. One might ask if the dose difference actually matters for an irreversibly binding proteasome inhibitor. Area under the plasma concentration-time curve seems to increase with dose from $20 \mathrm{mg} / \mathrm{m}^{2}$ to $56 \mathrm{mg} / \mathrm{m}^{2}$, with dose-dependent inhibition of proteasome activity, at least in peripheral blood cells. ${ }^{9}$ Further studies are therefore still needed to establish standard carfilzomib doses and administration schedules for combinations therapies for relapsed multiple myeloma. Meanwhile, physicians will need to rely on careful scrutiny of adverse event profiles in patient subgroups, as well as reports of real-world experience. Undoubtedly, though, the results of the A.R.R.O.W. study usher in a new era in carfilzomib therapy.

Holger W. Auner, Kwee Yong*

Department of Medicine, Imperial College London, London, UK (HWA); UCL Cancer Institute, University College London, Gower Street, London, WC1E 6BT, UK (KY)

kwee.yong@ucl.ac.uk

1. Moreau P, Pylypenko H, Grosicki S, et al. Subcutaneous versus intravenous administration of bortezomib in patients with relapsed multiple myeloma: a randomised, phase 3, non-inferiority study. Lancet Oncol 2011; 12(5): 431-40.

2. Moore S, Atwal S, Sachchithanantham S, et al. Weekly intravenous bortezomib is effective and well tolerated in relapsed/refractory myeloma. Eur J Haematol 2013; 90(5): 4205.

3. Mateos MV, Bringhen S, Richardson PG, et al. Bortezomib cumulative dose, efficacy, and tolerability with three different bortezomib-melphalan-prednisone regimens in previously untreated myeloma patients ineligible for high-dose therapy. Haematologica 2014; 99(6): 1114-22.

4. Stewart AK, Rajkumar SV, Dimopoulos MA, et al. Carfilzomib, lenalidomide, and dexamethasone for relapsed multiple myeloma. N Engl J Med 2015; 372(2): 142-52.

5. Dimopoulos MA, Goldschmidt H, Niesvizky R, et al. Carfilzomib or bortezomib in relapsed or refractory multiple myeloma (ENDEAVOR): an interim overall survival analysis of an open-label, randomised, phase 3 trial. Lancet Oncol 2017; 18(10): 1327-37.

6. Dimopoulos MA, Moreau P, Palumbo A, et al. Carfilzomib and dexamethasone versus bortezomib and dexamethasone for patients with relapsed or refractory multiple myeloma 
(ENDEAVOR): a randomised, phase 3, open-label, multicentre study. Lancet Oncol 2016; 17(1): 27-38.

7. Moreau PM, M.V; Berenson, J.R.; Weisel, K.; Lazzaro, A.;, Song, K.; Dimopoulos, M.A.; Huang, M.; Zahlten-Kumeli, A.; Stewart, K.A. Once-weekly versus twice-weekly carfilzomib dosing in patients with relapsed and refractory multiple myeloma: results of the randomized phase 3 study A.R.R.O.W. The Lancet Oncology 2018.

8. Berenson JR, Cartmell A, Bessudo A, et al. CHAMPION-1: a phase 1/2 study of onceweekly carfilzomib and dexamethasone for relapsed or refractory multiple myeloma. Blood 2016; 127(26): 3360-8.

9. Papadopoulos KP, Siegel DS, Vesole DH, et al. Phase I study of 30-minute infusion of carfilzomib as single agent or in combination with low-dose dexamethasone in patients with relapsed and/or refractory multiple myeloma. J Clin Oncol 2015; 33(7): 732-9. 